\title{
Improved Accuracy By Adapted Mesh-Refinements in the Finite Element Method
}

\author{
By Kenneth Eriksson
}

\begin{abstract}
For appropriately adapted mesh-refinements, optimal order error estimates are proved for the finite element approximate solution of the Neumann problem for the second-order elliptic equation $L u=\delta$, where $\delta$ is the Dirac distribution.
\end{abstract}

1. Introduction and Statement of Results. Let $\Omega$ be a bounded domain in $R^{N}$, $N \geqslant 2$, with smooth ( $C^{\infty}$ regular) boundary $\Gamma$. Given $x_{0} \in \bar{\Omega}$ we shall consider the Neumann problem

$$
\begin{gathered}
L u(x) \equiv-\sum_{i, j=1}^{N} \frac{\partial}{\partial x_{j}}\left(a_{i j}(x) \frac{\partial u}{\partial x_{i}}\right)+\sum_{i=1}^{N} a_{i}(x) \frac{\partial u}{\partial x_{i}}+a(x) u \\
=\delta\left(x-x_{0}\right) \quad \text { in } \Omega, \\
l u(x) \equiv \sum_{i, j=1}^{N} a_{i j}(x) \frac{\partial u}{\partial x_{i}} n_{j}=0 \text { on } \Gamma,
\end{gathered}
$$

where $a_{i j}, a_{i}$, and $a$ are smooth functions on $\bar{\Omega}$, where $\delta$ is the Dirac delta distribution (unit impulse), and where $n=\left(n_{j}\right)$ is the exterior normal to $\Gamma$.

For instance, Eq. (1.1a) models diffusion-type processes where the "source" (source of pollution, say) is located at a given point $x_{0}$. The particular choice of boundary conditions has been made merely for convenience. From a mathematical point of view, the solution of (1.1) is also the Green's function for the larger class of problems: $L u=f$ in $\Omega, l u=0$ on $\Gamma$.

We shall assume that the bilinear form

$$
A(u, v) \equiv \int_{\Omega}\left(\sum_{i, j} a_{i j} \frac{\partial u}{\partial x_{i}} \frac{\partial v}{\partial x_{j}}+\sum_{i} a_{i} \frac{\partial u}{\partial x_{i}} v+a u v\right) d x
$$

associated with $L$ satisfies the ellipticity-coercivity condition

$$
A(v, v) \geqslant c_{A}\|v\|_{1}^{2} \quad \forall v \in H^{1}, c_{A}>0,
$$

where $\|\cdot\|_{1}$ is the norm in the Sobolev space $H^{1}=H^{1}(\Omega)$. It is known (cf., e.g., [10, Theorem 3.3]) that problem (1.1) admits a unique solution $u$ with a singularity at $x_{0}$

Received September 28, 1982.

1980 Mathematics Subject Classification. Primary 65N15, 65N30.

Key words and phrases. Neumann problem, Green's function, finite element method, mesh-refinement, error estimate. 
of order $\left|x-x_{0}\right|^{-N+2}$ if $N>2$, and $\log \left(\left|x-x_{0}\right|^{-1}\right)$ if $N=2$. Away from $x_{0}$ the solution is smooth.

Noting that for sufficiently regular test functions $\psi$ the solution $u$ of (1.1) satisfies

$$
A(u, \psi)=\psi\left(x_{0}\right) \text {, }
$$

we define for any finite-dimensional subspace $S_{h}$ of $H^{1} \cap C(\bar{\Omega})$ the Galerkin approximate solution $u_{h} \in S_{h}$ by

$$
A\left(u_{h}, \chi\right)=\chi\left(x_{0}\right) \quad \forall \chi \in S_{h} .
$$

By the fact that $A(\cdot, \cdot)$ is positive there exists a unique such $u_{h}$.

Assuming standard finite element approximation properties of $S_{h}$, Babuška [2] was able to show, for $N=2$ and $L=-\Delta+I$ (minus Laplacian plus identity), that

$$
\left\|u_{h}-u\right\|_{0} \leqslant C_{\varepsilon} h^{1-\varepsilon}
$$

where $\|\cdot\|_{0}$ is the $L_{2}$-norm and $\varepsilon>0$ is arbitrary. Later, Scott [14] improved and generalized this result by showing that for general dimension $N \geqslant 2$, elliptic operators $L$ of order $2 m$, and normal covering boundary conditions,

$$
\left\|u_{h}-u\right\|_{s} \leqslant C\left(x_{0}\right) h^{2 m-s-N / 2} \text { for } 2 m-k \leqslant s<2 m-N / 2,
$$

where $k \geqslant 2 m$ is the order of approximation of $S_{h}$, where $C\left(x_{0}\right)$ tends to infinity as $x_{0}$ approaches $\Gamma$, and where the Sobolev norm index $s$ may also be negative. Results on discrete Green's functions and problems with nonsmooth right-hand sides can also be found in, e.g., [3], [5], [7], and [13].

In this paper we use finite element spaces $S_{h}$ on refined (graded) meshes, adapted to the known singularity of $u$, to derive optimal order global error estimates in the $L_{1}$-norm, and to show improved pointwise convergence near the singularity. As a corollary we find that when applied to a regular problem our method yields a superconvergent approximation of the true solution at $x_{0}$. We shall now describe our prerequisites and results in more detail.

For simplicity, we shall only consider finite element spaces consisting of piecewise polynomials on "simplicial" partitions of $\Omega$. More precisely, let $\Omega$ be divided into elements $\tau$ in such a way that each $\tau$ is the restriction to $\Omega$ of the interior of a simplex $\hat{\tau}$, and such that the intersection of any two such simplices is either a common face of both, or of lower dimension (cf., e.g., [6]). To enable a piecewise polynomial on such a partition to fit better the singularity of $u$ at $x_{0}$, we shall assume that the mesh-size is related to the distance to $x_{0}$ by

$$
C_{R}^{-1} \operatorname{diam}(\tau) \leqslant h\left(\operatorname{dist}\left(x_{0}, \tau\right)\right)^{\alpha}+h^{1 \wedge 1-\alpha)} \leqslant C_{R} \operatorname{diam}(\tau) \quad \forall \tau,
$$

where $C_{R}$ ( $R$ for refinement) is a given constant, $h \in\left(0, \frac{1}{2}\right]$ is the global mesh-size parameter, and $\alpha \in[0,1)$ determines the degree of refinement. Thus, elements on distance $d$ from $x_{0}$ have diameters of order $h d^{\alpha}$, and those closest to $x_{0}$ have diameters of order $h^{1} \wedge^{1-\alpha)}$. In order to have (local) inverse estimates we shall assume that

$$
(\operatorname{diam}(\hat{\tau}))^{N} \leqslant C_{S} m(\tau) \quad \forall \tau,
$$

where $m(\tau)$ denotes the measure $\int_{\tau} d x$ of $\tau$, and $C_{S}>0$ is a given constant. Hence the simplices $\hat{\tau}$ do not degenerate, and the elements $\tau$ are locally quasi-uniform. We shall denote by $\Delta_{h}=\Delta_{h}\left(x_{0}, \alpha\right)$ a partition of $\Omega$ obtained as above and satisfying 
(1.5) and (1.6), and by $\hat{\Omega}_{h}$ the corresponding union of simplices $\hat{\tau}$. For $S_{h}=$ $S_{h}\left(x_{0}, \alpha, r\right)$ we take the restrictions to $\bar{\Omega}$ of all continuous functions on $\hat{\Omega}_{h}$ which reduce to polynomials of degree at most $r-1$ on each simplex $\hat{\tau}$ of $\hat{\Omega}_{h}$.

Throughout the paper, $c$ and $C$, not necessarily the same at different places, and $C_{1}, C_{2}, C_{3}$, and $C_{*}$ will denote constants which may depend on $c_{A}, C_{R}, C_{S}, \alpha, r, N$, the diameter of $\Omega$, the smoothness of $\Gamma$, and the smoothness of the coefficients of $L$, but not on $h$ and $x_{0}$.

We are now ready to state our first results, with $\nabla$ denoting the gradient.

THEOREM 1. Let $u$ be the solution of (1.1) and $u_{h} \in S_{h}\left(x_{0}, \alpha, r\right)$ that of (1.4). Then, for $\alpha>(r-2) /(r-1)$,

$$
\left\|\nabla\left(u_{h}-u\right)\right\|_{L_{1}} \leqslant C h^{r-1}
$$

and for $\alpha>(r-2) / r$

$$
\left\|u_{h}-u\right\|_{L_{1}} \leqslant C h^{r}
$$

Remark. In the duality argument used in the proof of (1.8), we gain more than one power of $h$ near the singularity since the mesh is refined. As a consequence of this, we can derive (1.8) for a somewhat weaker refinement than (1.7).

Remark. We mention without proof that for $\alpha=(r-2) /(r-1)$ and $\alpha=$ $(r-2) / r$ there are logarithmic modifications of (1.7) and (1.8), respectively. For example, for $r=2$ and $\alpha=0$ (the quasi-uniform case) we have the well-known result (cf. [15] for a special case)

$$
\left\|\nabla\left(u_{h}-u\right)\right\|_{L_{1}} \leqslant C\left(\ln \frac{1}{h}\right) h .
$$

Our next result concerns the pointwise convergence and should be compared with Theorem 6.1 of [13] for quasi-uniform meshes.

THEOREM 2. Let $u$ and $u_{h}$ be as in Theorem 1 with $\alpha>(r-2) / r$. Let $\left|x-x_{0}\right|=d$ and $\operatorname{dist}(x, \Gamma) \geqslant d$, where $d \geqslant c h^{1 / 1-\alpha)}$ for a suitable $c>0$. Then

$$
\left|u_{h}(x)-u(x)\right| \leqslant C d^{-N}\left(\ln \frac{1}{h}\right)^{\bar{r}} h^{r}
$$

where $\bar{r}=1$ if $r=2, \bar{r}=0$ if $r>2$.

Remark. Here the restriction on $\operatorname{dist}(x, \Gamma)$ is put on only for the ease of reference in the proof. The necessary arguments for a proof in the general case can be found in [8].

Our estimate (1.7) has the following notable implication.

COROLlary. Let $v$ be the solution of

$$
\begin{aligned}
L^{*} v & \equiv-\sum_{i, j=1}^{N} \frac{\partial}{\partial x_{i}}\left(a_{i j} \frac{\partial v}{\partial x_{j}}\right)-\sum_{i=1}^{N} \frac{\partial}{\partial x_{i}}\left(a_{i} v\right)+a v=f \text { in } \Omega, \\
l^{*} v & \equiv \sum_{i, j=1}^{N} a_{i j} \frac{\partial v}{\partial x_{j}} n_{i}+\sum_{i=1}^{N} a_{i} v n_{i}=0 \quad \text { on } \Gamma,
\end{aligned}
$$

or in weak form, with $(\cdot, \cdot)$ denoting the $L_{2}(\Omega)$-inner product,

$$
A(\psi, v)=(\psi, f) \quad \forall \psi \in H^{1},
$$


and let $v_{h} \in S_{h}\left(x_{0}, \alpha, r\right)$ be the Galerkin approximate solution defined by

$$
A\left(\chi, v_{h}\right)=(\chi, f) \quad \forall \chi \in S_{h} .
$$

Then, for $\alpha>(r-2) /(r-1)$,

$$
\left|v_{h}\left(x_{0}\right)-v\left(x_{0}\right)\right| \leqslant C h^{2 r-2}\|v\|_{w_{\infty}^{r}},
$$

where the latter norm is defined below.

Remark. Actually, (1.10) holds also for smaller $\alpha$ 's, that is, with less degree of refinement than stated here. This, and further results in this direction, will be shown in a forthcoming paper.

The proof of the above theorems and corollary can be found in Section 5 below. Sections 2, 3, and 4 are devoted to preliminary work. In Section 6 we show that the number of elements in our considered refined partitions are of the same order, $O\left(h^{-N}\right)$, as in the corresponding quasi-uniform ones. Hence the amount of work in solving for the Galerkin approximate solution is not essentially increased when using our adapted finite element spaces $S_{h}\left(x_{0}, \alpha, r\right)$ instead of standard ones. We also show that mesh-refinements of the considered type, satisfying (1.5) and (1.6), can indeed be constructed. For completeness, a result on continuous extension used in Section 2 is proved in an appendix. This briefly describes the organization of the paper. We now close this section by introducing some notation.

Besides the usual $L_{p}$-spaces and norms, we shall use the Sobolev spaces $W_{p}^{k}\left(\Omega^{\prime}\right)$ with norms

$$
\|v\|_{W_{p}^{k}\left(\Omega^{\prime}\right)}=\left(\sum_{|\beta| \leqslant k}\left\|D^{\beta} v\right\|_{L_{p}\left(\Omega^{\prime}\right)}^{p}\right)^{1 / p}
$$

and seminorms

$$
|v|_{W_{p}^{k}\left(\Omega^{\prime}\right)}=\left(\sum_{|\beta|=k}\left\|D^{\beta} v\right\|_{L_{p}\left(\Omega^{\prime}\right)}^{p}\right)^{1 / p},
$$

where $|\beta|=\beta_{1}+\cdots+\beta_{N}$ is the length of the multi-index $\beta=\left(\beta_{1}, \ldots, \beta_{N}\right), \beta_{i} \geqslant 0$ for $1 \leqslant i \leqslant N$, and where

$$
D^{\beta}=\left(\frac{\partial}{\partial x_{1}}\right)^{\beta_{1}} \cdots\left(\frac{\partial}{\partial x_{N}}\right)^{\beta_{N}} .
$$

For $p=2$ we shall write $\|\cdot\|_{k, \Omega^{\prime}}$ and $|\cdot|_{k, \Omega^{\prime}}$ for these norms and seminorms, respectively, and $H^{k}\left(\Omega^{\prime}\right)$ for the corresponding space. Hence $\|\cdot\|_{0, \Omega^{\prime}}$ is the $L_{2}$-norm over $\Omega^{\prime}$. If no domain is specified, it is understood to be all of $\Omega$.

In the proofs we shall divide $\Omega$ into subdomains characterized by the distance to the point $x_{0}$, or domains defined by

$$
\Omega_{j}=\left\{x \in \Omega:\left|x-x_{0}\right|<2^{-j}\right\}, \quad \text { and } D_{j}=\left\{x \in \Omega: 2^{-(j+1)}<\left|x-x_{0}\right|<2^{-j}\right\} \text {. }
$$

Since $\Omega$ is bounded, there is an integer $j_{1}$ such that the $D_{j}$ 's are empty for $j<j_{1}$. We also define $d_{j}=2^{-j}$ and $h_{j}=h d_{j}^{\alpha}$. Thus $d_{j}$ is proportional to the diameter of $\Omega_{j}$ and $D_{j}$, and, as long as $j$ is not too large so that $h_{j}$ is smaller than the minimal mesh-size $h^{1 / 1-\alpha)}, h_{j}$ is proportional to the maximal mesh-size on $\Omega_{j}$ and $D_{j}$. We shall frequently use the obvious facts that $d_{j} \leqslant C d_{j+1}$ and $h_{j} \leqslant C h_{j+1}$. In the technical 
work below we shall also need to know that the local mesh-size on $D_{j}$ is "small" in relation to the dimensions of $D_{j}$ in the sense that $h_{j} d_{j}^{-1} \leqslant c$ for a suitable (small) constant $c$. This will be the case for all $j \leqslant J_{1}$ if we define $J_{1}$ by $2^{-J_{1}}=C_{1} h^{1 / 1-\alpha)}$ for a sufficiently large constant $C_{1}$, since then, for $\operatorname{such} j$,

$$
h_{j} d_{j}^{-1}=h d_{j}^{\alpha-1} \leqslant h d_{J_{1}}^{\alpha-1}=1 / C_{1}^{1-\alpha} .
$$

Further, in the proofs we shall set $D_{j}^{1}=\Omega_{j-1} \backslash \bar{\Omega}_{j+2}$, and fix an element $\tau_{0}$ such that $x_{0} \in \bar{\tau}_{0}$. Finally, $B_{d}(y)$ will denote the ball of radius $d$ centered at $y$, and $P_{k}(E)$ the space of polynomials of degree at most $k$ restricted to $E$.

2. Properties of $S_{h}\left(x_{0}, \alpha, r\right)$. Let $\Delta_{h}=\Delta_{h}\left(x_{0}, \alpha\right)$ and $S_{h}=S_{h}\left(x_{0}, \alpha, r\right)$ be as in Section 1, and recall, with an obvious method of notation, that each $\chi \in S_{h}$ is the restriction to $\bar{\Omega}$ of a piecewise polynomial $\hat{\chi} \in \hat{S}_{h}$, defined on $\hat{\Omega}_{h} \supset \bar{\Omega}$.

We begin by showing the following inverse property, which is well known for the interior elements.

LEMMA 1. For $0 \leqslant t \leqslant s \leqslant r-1,1 \leqslant p, q \leqslant \infty, \tau \in \Delta_{h}$, and $\chi \in S_{h}$ (or, $\chi \in$ $\left.P_{r-1}(\hat{\tau})\right)$,

$$
|\chi|_{W_{p}^{s}(\tau)} \leqslant C(\operatorname{diam}(\tau))^{-(s-t)-N(1 / q-1 / p)}|\chi|_{W_{q}^{t}(\tau)} .
$$

Proof. Let $T$ be the unit $N$-simplex defined by

$$
T=\left\{x \in R^{N}: x_{i} \geqslant 0 \text { for } 1 \leqslant i \leqslant N, \sum_{i=1}^{N} x_{i} \leqslant 1\right\} .
$$

In view of our assumption (1.6) there are constants $c$ and $C$ and for each element $\tau$ an affine transformation of variables $y=A_{\tau}(x)=B_{\tau} x+b_{\tau}$, where $B_{\tau}=\left(b_{i j}\right)$ is invertible with inverse $B_{\tau}^{\prime}=\left(b_{i j}^{\prime}\right)$, such that

$$
c T \subset A_{\tau}(\tau) \subset A_{\tau}(\hat{\tau})=T, \quad\left|b_{i j}\right| \leqslant C(\operatorname{diam}(\tau))^{-1},
$$

and $\left|b_{i j}^{\prime}\right| \leqslant C \operatorname{diam}(\tau)$.

Define the operator $a_{\tau}$ by

$$
a_{\tau} \chi(y)=\chi\left(A_{\tau}^{-1}(y)\right)=\chi(x) .
$$

Using the chain rule and the estimates on $b_{i j}$ and $b_{i j}^{\prime}$, we have

$$
\left|D_{x}^{\beta} \hat{\chi}(x)\right|^{p} \leqslant N^{|\beta| p}\left(C(\operatorname{diam}(\tau))^{-1}\right)^{|\beta| p} \sum_{|\gamma|=|\beta|}\left|D_{y}^{\gamma} a_{\tau} \hat{\chi}(y)\right|^{p}
$$

and

$$
\left|\operatorname{det}\left(B_{\tau}^{\prime}\right)\right| \leqslant N ! C^{N}(\operatorname{diam}(\tau))^{N}
$$

and hence

$$
|\chi|_{W_{p}^{s}(\tau)} \leqslant C(\operatorname{diam}(\tau))^{N / p-s}\left|a_{\tau} \hat{\chi}\right|_{W_{p}^{s}(T)}
$$

Similarly,

$$
\left|a_{\tau} \chi\right|_{W_{q}^{t}(c T)} \leqslant C(\operatorname{diam}(\tau))^{-N / q+t}|\chi|_{W_{q}^{t}(\tau)} .
$$

Finally, since $P_{r-1}(T)$ is finite dimensional we have the seminorm inequality

$$
\left|a_{\tau} \hat{\chi}\right|_{W_{p}^{s}(T)} \leqslant C\left|a_{\tau} \hat{\chi}\right|_{W_{q}^{t}(c T)},
$$

which completes the proof. 
We shall use the following consequence of Lemma 1 . Let $D_{h} \subset D_{j}^{1}, j \leqslant J_{1}$, where $D_{h}$ is a mesh-domain, that is, the interior of the closure of a union of elements. Then, for $\chi \in S_{h}$,

$$
\|\chi\|_{1, D_{h}} \leqslant C h_{j}^{-1}\|\chi\|_{0, D_{h}}
$$

where $h_{j}=h d_{j}^{\alpha}$ is the local mesh-size on $D_{j}^{1} \supset D_{h}$.

We now turn to the question of approximation properties of $S_{h}\left(x_{0}, \alpha, r\right)$ and shall first prove the following basic result.

LEMMA 2. For each element $\tau \in \Delta_{h}$ there is a neighborhood $O_{\tau}$ of $\tau$ and for each $v \in L_{1}$ an interpolant $v_{I} \in S_{h}$ of $v$, such that $\operatorname{diam}\left(O_{\tau}\right) \leqslant C \operatorname{diam}(\tau)$, and such that for $0 \leqslant l \leqslant m \leqslant r, 1 \leqslant p \leqslant \infty$, and $v \in W_{p}^{m}\left(O_{\tau}\right)$, the estimate

$$
\left\|v_{I}-v\right\|_{W_{p}^{\prime}(\tau)} \leqslant C(\operatorname{diam}(\tau))^{m-l}\|v\|_{W_{p}^{m}\left(O_{\tau}\right)}
$$

holds.

Proof. The definition of $v_{I}$ is complicated both by the fact that the boundary is curved and by the fact that a function in $L_{1}$ does not have well-defined point values. Hence, we shall first extend $v$ outside $\Omega$ with retained local regularity properties. We then choose a set $Q$ of interpolation points where given values determine a function in $\hat{S}_{h}$ uniquely. To each $z \in Q$ we assign a certain mean value of the now extended $v$ over a locally defined small neighborhood of $z$. Finally, we take as $v_{I}$ the restriction to $\bar{\Omega}$ of the function in $\hat{S}_{h}$ determined by these values. For this $v_{I}$ we then prove the asserted estimate.

(i) Extension of $v$. By Lemma $\mathrm{A}$ of the appendix there exists a linear extension operator $E: L_{1}(\Omega) \rightarrow L_{1}\left(R^{N}\right)$ and constants $C_{E}$ and $C$ such that for $x \in \bar{\Omega}, d>0$, $0 \leqslant k \leqslant r, 1 \leqslant p \leqslant \infty$, and $v \in W_{p}^{k}\left(B_{C_{E} d}(x) \cap \Omega\right)$,

$$
\|E v\|_{W_{p}^{k}\left(B_{d}(x)\right)} \leqslant C\|v\|_{W_{p}^{k}\left(B_{C_{E^{d}}}(x) \cap \Omega\right)} .
$$

(ii) Interpolation points. Let $A_{\tau}$ be as in the proof of Lemma 1 and set $Q_{\tau}=$ $A_{\tau}^{-1}\left(Q_{r}\right)$, where

$$
Q_{r}=\left\{x \in T: x_{i} \in\{j /(r-1): j=0, \ldots, r-1\}, i=1, \ldots, N, \sum_{i=1}^{N} x_{i} \leqslant 1\right\} .
$$

Since a polynomial in $P_{r-1}$ is determined by its values in $Q_{r}$ (cf. [6, Theorem 2.2.1]) and since $P_{r-1}$ is preserved under nonsingular affine transformations of the variables, it follows that given values in $Q_{\tau}$ determine a unique polynomial in $P_{r-1}$. Hence, given values in the set $Q=\cup_{\tau} Q_{\tau}$ determine a unique piecewise polynomial in $\hat{S}_{h}$.

(iii) Mean value functionals $M_{z}$. Set $O_{\tau}^{\prime}=A_{\tau}^{-1}\left(B_{2}(0)\right)$. This set is a forerunner to the neighborhood $O_{\tau}$ of $\tau$ mentioned in the lemma. In fact, for interior elements we shall take $O_{\tau}=O_{\tau}^{\prime}$. For $z \in Q$ let $O_{z}$ be the intersection of all $O_{\tau}^{\prime}$ for which $z \in \bar{\tau}$. We shall define $M_{z}: L_{1}\left(O_{z}\right) \rightarrow R$ as a linear mean value functional such that $M_{z} p=p(z)$ for $p \in P_{r-1}$. For this purpose, let $M$ be a (fixed) bounded function with support in $B_{1}(0)$, and with the property

$$
\int_{B_{1}(0)} M(y) p(y) d y=p(0) \quad \forall p \in P_{r-1} .
$$


Consult [9] for the construction of a smooth such $M$. Let $B_{\varepsilon}(z)$ be the ball of maximal radius contained in $O_{z}$. As a consequence of (1.6), $\varepsilon=\varepsilon(z)$ is of order $\operatorname{diam}(\tau)$ for each $\tau$ with $z \in \bar{\tau}$. Set

$$
M_{\varepsilon}(x)=\varepsilon^{-N} M((x-z) / \varepsilon)
$$

and define for $z \in Q$

$$
M_{z} f \equiv \int_{B_{\varepsilon}(z)} M_{\varepsilon}(x) f(x) d x
$$

By (2.5) there follows

$$
M_{z} p=p(z) \quad \forall p \in P_{r-1}, \forall z \in Q,
$$

and since $\sup \left|M_{\varepsilon}\right|=\varepsilon^{-N} \sup |M|$ we obtain

$$
\left|M_{z} f\right| \leqslant C(\operatorname{diam}(\tau))^{-N}\|f\|_{L_{1}\left(O_{z}\right)}
$$

with $C$ independent of $z, \tau$, and $f$.

We are now ready to define $v_{I}$. Given $v \in L_{1}$ and its extension $E v$, let $v_{I}$ be the restriction to $\bar{\Omega}$ of $\hat{v}_{I} \in \hat{S}_{h}$ determined by

$$
\hat{v}_{I}(z)=M_{z}(E v) \quad \forall z \in Q .
$$

With $v_{I}$ thus defined we shall now proceed to prove (2.3), using a technique similar to that in [4].

(iv) Functionals $F_{\tau}$. Let $\pi_{\tau}: L_{1}\left(O_{\tau}^{\prime}\right) \rightarrow P_{r-1}$ be defined by

$$
\pi_{\tau} f(z)=M_{z} f \quad \forall z \in Q_{\tau},
$$

and note that by property (2.6) of $M_{z}$

$$
\pi_{\tau} p=p \quad \forall p \in P_{r-1} .
$$

Let $a_{\tau}$ be as in the proof of Lemma 1 and $a_{\tau}^{-1}$ its inverse. Associate with each $\tau$ the sublinear functional $F_{\tau}$ on $W_{p}^{m}\left(B_{2}(0)\right)$ defined by

$$
F_{\tau}(w)=\left\|a_{\tau} \pi_{\tau} a_{\tau}^{-1} w-w\right\|_{w_{p}^{\prime}(T)} .
$$

We assert that

$$
F_{\tau}(p)=0 \quad \forall p \in P_{r-1}
$$

and that

$$
F_{\tau}(w) \leqslant C\|w\|_{W_{p}^{m}\left(B_{2}(0)\right)} .
$$

The first claim is obvious since for $p \in P_{r-1}$,

$$
a_{\tau} \pi_{\tau} a_{\tau}^{-1} p=a_{\tau} a_{\tau}^{-1} p=p
$$

For the boundedness of $F_{\tau}$, it is sufficient to show that

$$
\left\|a_{\tau} \pi_{\tau} a_{\tau}^{-1} w\right\|_{W_{p}^{\prime}(T)} \leqslant C\|w\|_{W_{p}^{m}\left(B_{2}(0)\right)} .
$$

Since $a_{\tau} \pi_{\tau} a_{\tau}^{-1} w \in P_{r-1}$, and since $\|\cdot\|_{L_{\infty}\left(Q_{r}\right)}$ and $\|\cdot\|_{W_{p}^{\prime}(T)}$ are equivalent norms on $P_{r-1}(T)$, we have for some $z \in \bar{\tau}$,

$$
\left\|a_{\tau} \pi_{\tau} a_{\tau}^{-1} w\right\|_{w_{p}^{\prime}(T)} \leqslant C\left\|a_{\tau} \pi_{\tau} a_{\tau}^{-1} w\right\|_{L_{\infty}\left(Q_{r}\right)}=C\left|a_{\tau} \pi_{\tau} a_{\tau}^{-1} w(z)\right|=C\left|M_{z} a_{\tau}^{-1} w\right| .
$$


To reach the desired estimate from here we use (2.7) and change variables,

$$
\left|M_{z} a_{\tau}^{-1} w\right| \leqslant C(\operatorname{diam}(\tau))^{-N}\left\|a_{\tau}^{-1} w\right\|_{L_{1}\left(O_{z}\right)} \leqslant C\|w\|_{L_{1}\left(B_{2}(0)\right)} \leqslant C\|w\|_{W_{p}^{m}\left(B_{2}(0)\right)} .
$$

Since $F_{\tau}$ is sublinear and vanishes on polynomials, we have

$$
F_{\tau}(w) \leqslant F_{\tau}(w-p)+F_{\tau}(p)=F_{\tau}(w-p) \quad \forall p \in P_{r-1},
$$

so that by the boundedness

$$
F_{\tau}(w) \leqslant C \inf _{p \in P_{r-1}}\|w-p\|_{W_{p}^{m}\left(B_{2}(0)\right)} .
$$

By the equivalence of the seminorms $\inf _{p \in P_{m-1}}\|\cdot-p\|_{W_{p}^{m}}$ and $|\cdot|_{W_{p}^{m}}$ (cf. Theorem 1 of [4]), we obtain

$$
F_{\tau}(w) \leqslant C|w|_{W_{p}^{m}\left(B_{2}(0)\right)}
$$

(v) Proof of (2.3). Let $v, E v, \hat{v}_{I}$, and $v_{I}$ be as above and note that $a_{\tau} \hat{v}_{I}=a_{\tau} \pi_{\tau} E v=$ $a_{\tau} \pi_{\tau} a_{\tau}^{-1} a_{\tau} E v$ on $T$. Hence

$$
\left\|a_{\tau} \hat{v}_{I}-a_{\tau} E v\right\|_{w_{p}^{\prime}(T)}=F_{\tau}\left(a_{\tau} E v\right) .
$$

Changing variables, we thus obtain

$$
\begin{aligned}
\left\|v_{I}-v\right\|_{W_{p}^{\prime}(\tau)} & \leqslant\left\|\hat{v}_{I}-E v\right\|_{W_{p}^{\prime}(\hat{\tau})} \\
& \leqslant C(\operatorname{diam}(\tau))^{N / p-I}\left\|a_{\tau} \hat{v}_{I}-a_{\tau} E v\right\|_{W_{p}^{\prime}(T)}=C(\operatorname{diam}(\tau))^{N / p-1} F_{\tau}\left(a_{\tau} E v\right) \\
& \leqslant C(\operatorname{diam}(\tau))^{N / p-l}\left|a_{\tau} E v\right|_{W_{p}^{m}\left(B_{2}(0)\right)} \leqslant C(\operatorname{diam}(\tau))^{m-I}\|E v\|_{W_{p}^{m}\left(O_{\tau}^{\prime}\right)} .
\end{aligned}
$$

In the case $O_{\tau}^{\prime} \subset \Omega$ this shows (2.3) with $O_{\tau}=O_{\tau}^{\prime}$ since $E v=v$ on $\Omega$. For elements near the boundary with some part of $O_{\tau}^{\prime}$ outside $\Omega$ we take $O_{\tau}=B_{C_{E} d^{\prime}}(x) \cap \Omega$, where $B_{d^{\prime}}(x)$ is the ball of smallest radius containing $O_{\tau}^{\prime}$ and with $x \in \bar{\Omega}$, and use (2.4). In both cases we have shown the desired estimate (2.3). The estimate of $\operatorname{diam}\left(O_{\tau}\right)$ is obvious by our definitions. This completes the proof.

We shall use the following consequences of Lemma 2. Let $D^{\prime} \subset D^{\prime \prime} \subset \Omega_{j-1}$ be such that $O_{\tau} \subset D^{\prime \prime}$ for each $\tau$ which intersects $D^{\prime}$. Then

$$
\left\|v_{I}-v\right\|_{l, D^{\prime}} \leqslant C h_{j}^{m-l}\|v\|_{m, D^{\prime \prime}} .
$$

For by assumption there is a mesh-domain $D_{h}$ such that $D^{\prime} \subset D_{h} \subset D^{\prime \prime}$ and with $O_{\tau} \subset D^{\prime \prime}$ for $\tau \subset D_{h}$, and hence

$$
\begin{aligned}
\left\|v_{I}-v\right\|_{l, D^{\prime}}^{2} & \leqslant\left\|v_{I}-v\right\|_{l, D_{h}}^{2}=\sum_{\tau \subset D_{h}}\left\|v_{I}-v\right\|_{l, \tau}^{2} \\
& \leqslant C h_{j}^{2(m-l)} \sum_{\tau \subset D_{h}}\|v\|_{m, O_{\tau}}^{2} \leqslant C h_{j}^{2(m-l)}\|v\|_{m, D^{\prime \prime}}^{2},
\end{aligned}
$$

where for the last step we have also used that at each point there is only a finite number of overlapping neighborhoods $O_{\tau}$. Further, we have of course the global estimate

$$
\left\|v_{I}-v\right\|_{W_{\infty}^{1}} \leqslant C h^{r-1}\|v\|_{W_{\infty}^{r}} .
$$

We end this section with a super-approximation result. 
LEMMA 3. Let $D_{h} \subset D_{j}^{1}$ be a mesh-domain and $\hat{D}_{h}$ the corresponding union of simplices. Further, let $\eta$ be a smooth function which is constant outside $\hat{D}_{h}$ (on each component of $\left.\operatorname{int}\left(\hat{\Omega}_{h}\right) \backslash \hat{D}_{h}\right)$ and satisfying

$$
\|\eta\|_{W_{\infty}^{k}\left(\Omega_{h}\right)} \leqslant \mu d_{j}^{-k}, \quad 0 \leqslant k \leqslant r .
$$

Then there is a constant $C_{\mu}>0$ and for each given $v_{h} \in S_{h}$ an interpolant $\zeta \in S_{h}$ of $\eta v_{h}$ such that $\operatorname{supp}\left(\eta v_{h}-\zeta\right) \subset \bar{D}_{h}$, and

$$
\left\|\eta v_{h}-\zeta\right\|_{1, D_{h}} \leqslant C_{\mu}\left(h_{j} d_{j}^{-1}\left\|v_{h}\right\|_{1, D_{h}}+h_{j} d_{j}^{-2}\left\|v_{h}\right\|_{0, D_{h}}\right) \text {. }
$$

Proof. In contrast to the situation in Lemma 2, the function $\eta v_{h}$ which is to be approximated is now continuous. Hence, we take as $\zeta$ the restriction to $\bar{\Omega}$ of $\hat{\zeta} \in \hat{S}_{h}$ defined by

$$
\hat{\zeta}(z)=\left(\eta \hat{v}_{h}\right)(z) \quad \forall z \in Q .
$$

Obviously, $\operatorname{supp}\left(\eta v_{h}-\zeta\right) \subset \bar{D}_{h}$, and the desired estimate over $D_{h}$ follows easily if we prove the corresponding estimate on each individual $\tau \subset D_{h}$.

For this, let $F$ be the functional on $W_{\infty}^{r}(T)$ defined by

$$
F(w)=\left\|w-\Pi_{T} w\right\|_{1, T}
$$

where $\Pi_{T} w \in P_{r-1}$ is determined by

$$
\Pi_{T} w(z)=w(z) \quad \forall z \in Q_{r} .
$$

Clearly, $F$ is bounded on $W_{\infty}^{r}(T)$ and vanishes on $P_{r-1}$. Hence, as in the proof of Lemma 2,

$$
F(w) \leqslant C|w|_{W_{\infty}^{r}(T)} \quad \forall w \in W_{\infty}^{r}(T) .
$$

With $a_{\tau}$ as before, note that by our definitions,

$$
F\left(a_{\tau}\left(\eta \hat{v}_{h}\right)\right)=\left\|a_{\tau}\left(\eta \hat{v}_{h}\right)-a_{\tau} \zeta\right\|_{1, T},
$$

and hence, changing variables,

$$
\begin{aligned}
\left\|\eta v_{h}-\zeta\right\|_{1, \tau} & \leqslant C(\operatorname{diam}(\tau))^{N / 2-1}\left\|a_{\tau}\left(\eta \hat{v}_{h}\right)-a_{\tau} \hat{\zeta}\right\|_{1, T} \\
& =C(\operatorname{diam}(\tau))^{N / 2-1} F\left(a_{\tau}\left(\eta \hat{v}_{h}\right)\right) \leqslant C(\operatorname{diam}(\tau))^{N / 2-1}\left|a_{\tau}\left(\eta \hat{v}_{h}\right)\right|_{W_{\infty}^{r}(T)} .
\end{aligned}
$$

We next use seminorm inequalities on $P_{r-1}(T)$ together with the fact that any $r$ th derivative of $\left.a_{\tau} \hat{v}_{h}\right|_{T} \in P_{r-1}(T)$ vanishes to obtain (with $c$ as in (2.1))

$$
\begin{aligned}
\left|a_{\tau}\left(\eta \hat{v}_{h}\right)\right|_{W_{\infty}^{r}(T)} & =\left|a_{\tau} \eta a_{\tau} \hat{v}_{h}\right|_{W_{\infty}^{r}(T)} \leqslant \sum_{k=1}^{r}\left|a_{\tau} \eta\right|_{W_{\infty}^{k}(T)}\left|a_{\tau} \hat{v}_{h}\right|_{W_{\infty}^{r-k}(T)} \\
& \leqslant C \sum_{k=1}^{r-1}\left|a_{\tau} \eta\right|_{W_{\infty}^{k}(T)}\left|a_{\tau} v_{h}\right|_{1, c T}+C\left|a_{\tau} \eta\right|_{W_{\infty}^{r}(T)}\left\|a_{\tau} v_{h}\right\|_{0, c T},
\end{aligned}
$$

so that returning to the original variables,

$$
\begin{aligned}
\left|a_{\tau}\left(\eta \hat{v}_{h}\right)\right|_{w_{\infty}^{r}(T)} \leqslant & C\left\|v_{h}\right\|_{1, \tau} \sum_{k=1}^{r-1}\|\eta\|_{W_{\infty}^{k}\left(\hat{\Omega}_{h}\right)}(\operatorname{diam}(\tau))^{k+1-N / 2} \\
& +C(\operatorname{diam}(\tau))^{r-N / 2} d_{j}^{-r}\left|v_{h}\right|_{0, \tau}
\end{aligned}
$$


or altogether,

$$
\left|\eta v_{h}-\zeta\right|_{1, \tau} \leqslant C\left\|v_{h}\right\|_{1, \tau} \sum_{k=1}^{r-1}|\eta|_{w_{\infty}^{k}\left(\hat{\Omega}_{h}\right)}(\operatorname{diam}(\tau))^{k}+C(\operatorname{diam}(\tau))^{r-1} d_{j}^{-r}\left\|v_{h}\right\|_{0, \tau}
$$

The desired estimate now follows by the assumptions on $\eta$ and the obvious estimates

$$
\operatorname{diam}(\tau) \leqslant C h_{j} \text { and } h_{j} d_{j}^{-1} \leqslant C,
$$

which hold since $\tau \subset D_{h} \subset D_{j}^{1}$. This completes the proof of Lemma 3 .

3. Elliptic Regularity. In this section we shall first recall some well-known elliptic theory. We then introduce an approximate Dirac delta distribution and the corresponding approximate solution $\tilde{u}$ of (1.1), and derive estimates by which the proof Theorem 1 may be reduced to estimating $u_{h}-\tilde{u}$.

Lemma 4. Let the bilinear form $A(\cdot, \cdot)$ and the operators $L$ and $l$ be as in Section 1. Then, given $f \in L_{2}$ there is a unique $v \in H^{1}$ such that

$$
A(v, \psi)=(f, \psi) \quad \forall \psi \in H^{1} .
$$

Actually, $v \in H^{2}$ and

$$
\|v\|_{2} \leqslant C\|f\|_{0}
$$

and $v$ is the unique solution of $L v=f$ in $\Omega, l v=0$ on $\Gamma$. Moreover, there is a function $g(x, y)$, the Green's function, which is smooth off the diagonal $x=y$, and such that

$$
\left|D_{x}^{\beta} D_{y}^{\gamma} g(x, y)\right| \leqslant \begin{cases}C|x-y|^{-N+2-|\beta|-|\gamma|} & \text { if }-N+2-|\beta|-|\gamma|<0 \\ C\left(\ln \left(|x-y|^{-1}\right)+1\right) & \text { if } N=2,|\beta|=|\gamma|=0\end{cases}
$$

and

$$
v(x)=\int_{\Omega} g(x, y) f(y) d y \quad(\text { a.e. }) \text { in } \Omega .
$$

Similarly, there are functions $v$ and $g$ (in general different from the above $v$ and $g$ ) such that

$$
A(\psi, v)=(\psi, f) \quad \forall \psi \in H^{1}
$$

and for which (3.2) through (3.4) hold.

These facts are all well known. The existence of unique $H^{1}$-solutions of (3.1) and (3.5) is guaranteed by the Lax-Milgram lemma, which can be found in e.g. [6]. The $H^{2}$-regularity is a special case of more general regularity estimates presented in [11]. For the existence of Green's functions we refer to [10].

We shall use the following consequences of Lemma 4: Let $v$ be the solution of (3.1) or (3.5) with $\operatorname{supp}(f) \subset \bar{\tau}_{0}$, and set $d_{x}=\operatorname{dist}\left(x, \tau_{0}\right)$. It follows from (3.4) and (3.3) that

$$
\left|D^{\beta} v(x)\right|=\left|\int_{\tau_{0}} D_{x}^{\beta} g(x, y) f(y) d y\right| \leqslant C d_{x}^{-N+2-|\beta|}\|f\|_{L_{1}},
$$

and hence, for $j \leqslant J_{1}$,

$$
\|v\|_{r, D_{j}} \leqslant C d_{j}^{N / 2}\|v\|_{W_{\infty}^{r}\left(D_{j}\right)} \leqslant C d_{j}^{-N / 2+2-r}\|f\|_{L_{1}} .
$$


Similarly, for $i \leqslant j$ and $f$ with support in $\bar{\Omega}_{j}$ we obtain (using (3.2) for $i=j-1$ and $i=j)$

$$
\|v\|_{2, D_{i}} \leqslant C d_{i}^{-N / 2} d_{j}^{N / 2}\|f\|_{0, \Omega_{j}}
$$

and for $f$ with support in $\bar{D}_{i}$

$$
\|v\|_{2, \Omega_{j}} \leqslant C d_{j}^{-N / 2} d_{i}^{N / 2}\|f\|_{0, D_{i}} .
$$

Following our program, we shall now define an approximate delta distribution $\tilde{\delta}$ and the corresponding approximate solution $\tilde{u}$ of (1.1) in such a way that the discrete solution $u_{h}$ of (1.1) is also the Galerkin approximation of $\tilde{u}$. For this purpose, recall that $x_{0} \in \bar{\tau}_{0}$ and let $\tilde{\delta}$ be the $L_{2}$-projection of $\delta$ onto $P_{r-1}\left(\bar{\tau}_{0}\right)$, and extend $\tilde{\delta}$ by zero outside $\bar{\tau}_{0}$ so that

$$
(\tilde{\delta}, \chi)=\chi\left(x_{0}\right) \quad \forall \chi \in S_{h}
$$

Further, let $\tilde{u}$ be the solution of

$$
\begin{array}{ll}
L \tilde{u}=\tilde{\delta} & \text { in } \Omega, \\
l \tilde{u}=0 & \text { on } \Gamma .
\end{array}
$$

Since $\chi=\tilde{\delta}$ on $\tau_{0}$ for some $\chi \in S_{h}$ (note that $\tilde{\delta} \notin S_{h}$ ), we obtain by (3.9) $\|\tilde{\delta}\|_{0}^{2}=\tilde{\delta}\left(x_{0}\right)$, so that by Lemma 1 ,

$$
\|\tilde{\delta}\|_{0}^{2} \leqslant C\left(\operatorname{diam}\left(\tau_{0}\right)\right)^{-N / 2}\|\tilde{\delta}\|_{0},
$$

or

$$
\|\tilde{\delta}\|_{0} \leqslant C\left(\operatorname{diam}\left(\tau_{0}\right)\right)^{-N / 2}
$$

and hence

$$
\|\tilde{\delta}\|_{L_{1}} \leqslant C \text {. }
$$

Setting $\varepsilon=\operatorname{diam}\left(\tau_{0}\right)$, so that $\tau_{0} \subset B_{\varepsilon}\left(x_{0}\right)$, we shall now estimate $\tilde{u}-u$ in terms of $\varepsilon$.

LEMMA 5. Let $u$ be the solution of (1.1) and $\tilde{u}$ that of (3.10). For $\varepsilon \in\left(0, \frac{1}{2}\right]$ we then have

$$
\|\nabla(\tilde{u}-u)\|_{L_{1}} \leqslant C \varepsilon, \text { and }\|\tilde{u}-u\|_{L_{1}} \leqslant C \varepsilon^{2} \ln \frac{1}{\varepsilon} .
$$

Proof. Set $\tilde{e}=\tilde{u}-u$ and note that

$$
\left\|\nabla^{i} \tilde{e}\right\|_{L_{1}} \leqslant\left\|\nabla^{i} u\right\|_{L_{1}\left(B_{2 \ell}\left(x_{0}\right)\right)}+\left\|\nabla^{i} \tilde{u}\right\|_{L_{1}\left(B_{2 \varepsilon}\left(x_{0}\right)\right)}+\left\|\nabla^{i} \tilde{e}\right\|_{L_{1}\left(\Omega \backslash B_{2 \epsilon}\left(x_{0}\right)\right)} \quad \text { for } i=0,1 .
$$

Since $u(\cdot)=g\left(\cdot, x_{0}\right)$, where $g$ is the Green's function, we have by (3.3), with $R=\left|x-x_{0}\right|$

$$
\left\|\nabla^{i} u\right\|_{L_{1}\left(B_{2 \varepsilon}\left(x_{0}\right)\right)} \leqslant C \int_{0}^{2 \varepsilon} R^{1-i}\left(\ln \frac{1}{R}+1\right)^{\sigma} d R \leqslant C \varepsilon^{2-i}\left(\ln \frac{1}{\varepsilon}\right)^{\sigma} \text { for } i=0,1,
$$

where $\sigma=1$ if $N=2$ and $i=0$, and $\sigma=0$ otherwise. Similarly,

$$
\tilde{u}(x)=\int_{\tau_{0}} g(x, y) \tilde{\delta}(y) d y,
$$


so that employing also Lemma 1 and (3.12) we find

$$
\left|\nabla^{i} \tilde{u}(x)\right| \leqslant\left\|\nabla_{x}^{i} g(x, \cdot)\right\|_{L_{1}\left(\tau_{0}\right)}\|\tilde{\delta}\|_{L_{\infty}\left(\tau_{0}\right)} \leqslant C \varepsilon^{2-i}(\ln 1 / \varepsilon)^{\sigma} \varepsilon^{-N}
$$

and hence,

$$
\left\|\nabla^{i} \tilde{u}\right\|_{L_{1}\left(B_{2 \varepsilon}\left(x_{0}\right)\right)} \leqslant C \varepsilon^{2-i}(\ln 1 / \varepsilon)^{\sigma} \quad \text { for } i=0,1 .
$$

It remains to estimate $\tilde{e}=\tilde{u}-u$ on $\Omega \backslash B_{2 \varepsilon}\left(x_{0}\right)$.

By a Taylor expansion

$$
\begin{aligned}
g(x, y)= & g\left(x, x_{0}\right)+\left(y-x_{0}\right) \cdot \nabla_{y} g\left(x, x_{0}\right)^{t} \\
& +\frac{1}{2}\left(y-x_{0}\right) \cdot \nabla_{y}^{2} g(x, \xi) \cdot\left(y-x_{0}\right)^{t}
\end{aligned}
$$

where $\xi=\xi(y)=\theta x_{0}+(1-\theta) y, 0 \leqslant \theta \leqslant 1$, and where $t$ denotes transpose. Since

$$
\tilde{e}(x)=\int_{\tau_{0}}\left(g(x, y)-g\left(x, x_{0}\right)\right) \tilde{\delta}(y) d y,
$$

(note that $\int_{\tau_{0}} \tilde{\delta}(y) d y=1$ ) and since

$$
\int_{\tau_{0}} \tilde{\delta} p d y=0 \quad \forall p \in P_{1}\left(\subset P_{r-1}\right) \text { such that } p\left(x_{0}\right)=0,
$$

we obtain

$$
\tilde{e}(x)=\int_{\tau_{0}}\left(y-x_{0}\right) \cdot \nabla_{y}^{2} g(x, \xi(y)) \cdot\left(y-x_{0}\right)^{t} \tilde{\delta}(y) d y .
$$

Therefore, using (3.3) and (3.12),

$$
|\tilde{e}(x)| \leqslant C \varepsilon^{2} \sup _{y \in \tau_{0}}\left|\nabla_{y}^{2} g(x, y)\right| \leqslant C \varepsilon^{2}\left|x-x_{0}\right|^{-N} \quad \text { for } x \in \Omega \backslash B_{2 \varepsilon}\left(x_{0}\right) .
$$

Similarly, by a Taylor expansion for $\nabla_{x} g(x, y)$, we obtain

$$
|\nabla \tilde{e}(x)| \leqslant C \varepsilon^{2} \sup _{y \in \tau_{0}}\left|\nabla_{y}^{2} \nabla_{x} g(x, y)\right| \leqslant C \varepsilon^{2}\left|x-x_{0}\right|^{-N-1} \quad \text { for } x \in \Omega \backslash B_{2 \varepsilon}\left(x_{0}\right) .
$$

Hence

$$
\|\tilde{e}\|_{L_{1}\left(\Omega \backslash B_{2 \varepsilon}\left(x_{0}\right)\right)} \leqslant C \varepsilon^{2} \ln 1 / \varepsilon
$$

and

$$
\|\nabla \tilde{e}\|_{L_{1}\left(\Omega \backslash B_{2 \varepsilon}\left(x_{0}\right)\right)} \leqslant C \varepsilon .
$$

Together, our estimates now yield the proof.

4. Local Estimates. Below we shall derive local $H^{1}$-error estimates similar to the interior estimates of Nitsche and Schatz [12]. But first we shall prove the following global estimate.

LEMMA 6. Let $f \in L_{2}$ have support in $\bar{\Omega}_{j}, j \leqslant J_{1}$, and let $v$ be the solution of (3.1) (or (3.5)) and $v_{h} \in S_{h}=S_{h}\left(x_{0}, \alpha, r\right)$ that of

$$
A\left(v_{h}, \chi\right)=(f, \chi) \quad\left(\text { or } A\left(\chi, v_{h}\right)=(\chi, f)\right) \quad \forall \chi \in S_{h} .
$$

Then, $\left\|v_{h}-v\right\|_{1} \leqslant C h_{j}\|f\|_{0}$. 
Proof. Using the coercivity and continuity of $A(\cdot, \cdot)$ together with the orthogonality with respect to $A(\cdot, \cdot)$ of $v_{h}-v$ and $S_{h}$, we have by standard arguments

$$
\left\|v_{h}-v\right\|_{1}^{2} \leqslant C\|v-\chi\|_{1}^{2} \quad \forall \chi \in S_{h} .
$$

Since

$$
\|\cdot\|_{1}^{2}=\|\cdot\|_{1, \Omega_{j}}^{2}+\sum_{i<j}\|\cdot\|_{1, D_{i}}^{2}
$$

we have, thus, in view of (2.8),

$$
\left\|v_{h}-v\right\|_{1}^{2} \leqslant C\left(h_{j}^{2}\|v\|_{2, \Omega_{j-1}}^{2}+\sum_{i<j} h_{i}^{2}\|v\|_{2, D_{i}^{1}}^{2}\right) \leqslant C\left(h_{j}^{2}\|v\|_{2}^{2}+\sum_{i \leqslant j} h_{i}^{2}\|v\|_{2, D_{i}}^{2}\right) .
$$

Using the elliptic regularity estimates (3.2) and (3.7), we obtain

$$
\left\|v_{h}-v\right\|_{1}^{2} \leqslant C\left(h_{j}^{2}+d_{j}^{N} \sum_{i \leqslant j} h_{i}^{2} d_{i}^{-N}\right)\|f\|_{0}^{2}
$$

and since $\alpha<1$ and $N \geqslant 2$,

$$
\begin{aligned}
\sum_{i \leqslant j} h_{i}^{2} d_{i}^{-N} & =h^{2} \sum_{i \leqslant j} d_{i}^{2 \alpha-N}=h^{2} \sum_{i \leqslant j} 2^{-i(2 \alpha-N)} \\
& =h^{2} 2^{-j(2 \alpha-N)} /\left(1-2^{2 \alpha-N}\right) \leqslant C h_{j}^{2} d_{j}^{-N} .
\end{aligned}
$$

This completes the proof of Lemma 6.

We now turn to the local estimates.

$$
\begin{aligned}
& \text { LemMa 7. Let } j \leqslant J_{1} \text { and let } v \in H^{r}\left(D_{j}^{1}\right) \text { and } v_{h} \in S_{h} \text { satisfy } \\
& \qquad A\left(v_{h}-v, \chi\right)=0 \quad \forall \chi \in S_{h} \text { with support in } D_{j}^{1} .
\end{aligned}
$$

Then,

$$
\left\|v_{h}-v\right\|_{1, D_{j}} \leqslant C\left(h_{j}^{r-1}\|v\|_{r, D_{j}^{1}}+d_{j}^{-1}\left\|v_{h}-v\right\|_{0, D_{j}^{1}}\right) .
$$

Proof. Let $D_{j} \subset D_{h} \subset D_{h}^{\prime} \subset D \subset D_{j}^{1}$, where $D_{h}$ and $D_{h}^{\prime}$ are mesh-domains, and let $\eta, \eta^{\prime}$, and $\omega$ be smooth cut-off functions such that

$$
\begin{aligned}
& \eta=1 \quad \text { on } D_{j}, \quad \eta=0 \quad \text { on } \hat{\Omega}_{h} \backslash \hat{D}_{h}, \quad\|\eta\|_{W_{\infty}^{k}\left(\hat{\Omega}_{h}\right)} \leqslant C d_{j}^{-k} \quad \text { for } 0 \leqslant k \leqslant r, \\
& \eta^{\prime}=1 \quad \text { on } D_{h}, \quad \eta^{\prime}=0 \quad \text { on } \hat{\Omega}_{h} \backslash \hat{D}_{h}^{\prime}, \quad\left\|\eta^{\prime}\right\|_{W_{\infty}^{k}\left(\hat{\Omega}_{h}\right)} \leqslant C d_{j}^{-k} \quad \text { for } 0 \leqslant k \leqslant r \text {, } \\
& \omega=1 \quad \text { on } D_{h}^{\prime}, \quad \omega=0 \quad \text { on } \Omega \backslash D, \quad\|\omega\|_{\omega_{\infty}^{k}(\Omega)} \leqslant C d_{j}^{-k} \quad \text { for } k=0,1,
\end{aligned}
$$

and such that if $\tau \cap D \neq \varnothing$ then $O_{\tau} \subset D_{j}^{1}$, where $O_{\tau}$ is the neighborhood of $\tau$ defined in Lemma 2. Note that this construction requires that the local mesh-size on $D_{j}^{1} \backslash D_{j}$ is sufficiently "small" which is the case for $j \leqslant J_{1}$, since $J_{1}$ was chosen not too large.

In order to prove the desired estimate, it is sufficient to show that

$$
\left\|v_{h}-v\right\|_{1, D_{j}} \leqslant C\left(\|v\|_{1, D}+d_{j}^{-1}\|v\|_{0, D}+d_{j}^{-1}\left\|v_{h}-v\right\|_{0, D}\right),
$$

since then, by replacing $v$ by $v_{I}-v$ and $v_{h}$ by $v_{I}-v_{h}$ and using $h_{j} d_{j}^{-1} \leqslant C$, it follows from (2.8) that

$$
\begin{aligned}
\left\|v_{h}-v\right\|_{1, D_{j}} & \leqslant C\left(\left\|v_{I}-v\right\|_{1, D}+d_{j}^{-1}\left\|v_{I}-v\right\|_{0, D}+d_{j}^{-1}\left\|v_{h}-v\right\|_{0, D}\right) \\
& \leqslant C\left(h_{j}^{r-1}\|v\|_{r, D_{j}^{1}}+d_{j}^{-1}\left\|v_{h}-v\right\|_{0, D_{j}^{1}}\right) .
\end{aligned}
$$


In order to prove (4.1), we have first

$$
\left\|v_{h}-v\right\|_{1, D_{j}} \leqslant\left\|v_{h}-(\omega v)_{h}\right\|_{1, D_{j}}+\left\|(\omega v)_{h}-\omega v\right\|_{1, D_{j}}
$$

where

$$
A\left((\omega v)_{h}-\omega v, \chi\right)=0 \quad \forall \chi \in S_{h}
$$

defines $(\omega v)_{h} \in S_{h}$. By the $H^{1}$-stability of the projection $(\cdot)_{h}$ and the properties of $\omega$, we have

$$
\left\|(\omega v)_{h}-\omega v\right\|_{1} \leqslant C\|\omega v\|_{1} \leqslant C\left(\|v\|_{1, D}+d_{j}^{-1}\|v\|_{0, D}\right) .
$$

It thus remains to estimate the term $\left\|v_{h}-(\omega v)_{h}\right\|_{1, D_{j}}$.

We shall show below that if $w_{h} \in S_{h}$ and

$$
A\left(w_{h}, \chi\right)=0 \quad \forall \chi \in S_{h} \text { with support in } \overline{D_{h}^{\prime}},
$$

$$
\left\|w_{h}\right\|_{1, D_{j}} \leqslant C d_{j}^{-1}\left\|w_{h}\right\|_{0, D_{h}^{\prime}} .
$$

Since (4.2) holds for $w_{h}=v_{h}-(\omega v)_{h} \in S_{h}$, we obtain from (4.3)

$$
\begin{aligned}
\left\|v_{h}-(\omega v)_{h}\right\|_{1, D} & \leqslant C d_{j}^{-1}\left\|v_{h}-(\omega v)_{h}\right\|_{0, D_{h}^{\prime}} \\
& \leqslant C d_{j}^{-1}\left\|v_{h}-v\right\|_{0, D_{h}^{\prime}}+C d_{j}^{-1}\left\|\omega v-(\omega v)_{h}\right\|_{0, D_{h}^{\prime}},
\end{aligned}
$$

where the last term still has to be estimated. For this purpose, we shall use a duality argument and show

$$
\left\|\omega v-(\omega v)_{h}\right\|_{0, D_{h}^{\prime}} \leqslant C h_{j}\|\omega v\|_{1},
$$

from which the desired estimate follows easily. It thus remains to prove (4.3) and (4.4).

For the proof of (4.4), let $\Phi \in H^{1}$ and $\Phi_{h} \in S_{h}$ be defined by

$$
A(\psi, \Phi)=(\psi, \phi) \quad \forall \psi \in H^{1}, \quad \text { and } A\left(\chi, \Phi_{h}\right)=(\chi, \phi) \quad \forall \chi \in S_{h},
$$

where $\phi \in L_{2}$ and $\operatorname{supp}(\phi) \subset D_{h}^{\prime}$. By our definitions and by Lemma 6 we then have

$$
\begin{aligned}
\left(\omega v-(\omega v)_{h}, \phi\right) & =A\left(\omega v(\omega v)_{h}, \Phi\right)=A\left(\omega v-(\omega v)_{h}, \Phi-\Phi_{h}\right) \\
& =A\left(\omega v, \Phi-\Phi_{h}\right) \leqslant C\|\omega v\|_{1}\left\|\Phi-\Phi_{h}\right\|_{1} \leqslant C\|\omega v\|_{1} h_{j}\|\phi\|_{0, D_{h}^{\prime}} .
\end{aligned}
$$

Since $\phi \in L_{2}\left(D_{h}^{\prime}\right)$ was arbitrary, (4.4) follows by duality.

In order to show that (4.2) implies (4.3) we shall use the super-approximation property. With $\eta$ as above we have by the coercivity

$$
\left\|w_{h}\right\|_{1, D_{1}}^{2} \leqslant\left\|\eta w_{h}\right\|_{1}^{2} \leqslant \frac{1}{c_{A}} A\left(\eta w_{h}, \eta w_{h}\right) .
$$

Further,

$$
\begin{aligned}
A\left(\eta w_{h}, \eta w_{h}\right)= & A\left(w_{h}, \eta^{2} w_{h}\right)+\int_{\Omega}\left(\sum_{i, j} a_{i j} \frac{\partial \eta}{\partial x_{i}} w_{h} \frac{\partial\left(\eta w_{h}\right)}{\partial x_{j}}\right) d x \\
& -\int_{\Omega}\left(\sum_{i, j} a_{i j} \frac{\partial\left(\eta w_{h}\right)}{\partial x_{i}} w_{h} \frac{\partial \eta}{\partial x_{j}}\right) d x+\int_{\Omega}\left(\sum_{i, j} a_{i j} w_{h}^{2} \frac{\partial \eta}{\partial x_{i}} \frac{\partial \eta}{\partial x_{j}}\right) d x \\
& +\int_{\Omega}\left(\sum_{i} a_{i} w_{h}^{2} \frac{\partial \eta}{\partial x_{i}}\right) d x
\end{aligned}
$$


where the last four integrals can be estimated by

$$
C\left(d_{j}^{-1}\left\|w_{h}\right\|_{0, D_{h}}\left\|\eta w_{h}\right\|_{1}+d_{j}^{-2}\left\|w_{h}\right\|_{0, D_{h}}^{2}\right) \leqslant \frac{1}{2} c_{A}\left\|\eta w_{h}\right\|_{1}^{2}+C d_{j}^{-2}\left\|w_{h}\right\|_{0, D_{h}}^{2} .
$$

Altogether, this shows that

$$
\left\|w_{h}\right\|_{1, D_{j}}^{2} \leqslant C A\left(w_{h}, \eta^{2} w_{h}\right)+C d_{j}^{-2}\left\|w_{h}\right\|_{0, D_{h}}^{2} .
$$

In view of (4.2) and Lemma 3 we may subtract a suitable $\zeta \in S_{h}$ from $\eta^{2} w_{h}$ and hence obtain

$$
\begin{aligned}
A\left(w_{h}, \eta^{2} w_{h}\right) & =A\left(w_{h}, \eta^{2} w_{h}-\zeta\right) \\
& \leqslant C\left\|w_{h}\right\|_{1, D_{h}}\left(h_{j} d_{j}^{-1}\left\|w_{h}\right\|_{1, D_{h}}+h_{j} d_{j}^{-2}\left\|w_{h}\right\|_{0, D_{h}}\right) \\
& \leqslant C\left(h_{j} d_{j}^{-1}\left\|w_{h}\right\|_{1, D_{h}}^{2}+d_{j}^{-2}\left\|w_{h}\right\|_{0, D_{h}}^{2}\right),
\end{aligned}
$$

where again we have used the fact that $h_{j} d_{j}^{-1} \leqslant C$. We have thus shown that

$$
\left\|w_{h}\right\|_{1, D_{j}}^{2} \leqslant C\left(h_{j} d_{j}^{-1}\left\|w_{h}\right\|_{1, D_{h}}^{2}+d_{j}^{-2}\left\|w_{h}\right\|_{0, D_{h}}^{2}\right) \text {. }
$$

Repeating the above arguments on $\left\|w_{h}\right\|_{1, D_{h}}^{2}$, we obtain

$$
\begin{aligned}
\left\|w_{h}\right\|_{1, D_{j}}^{2} & \leqslant C h_{j} d_{j}^{-1}\left(h_{j} d_{j}^{-1}\left\|w_{h}\right\|_{1, D_{h}^{\prime}}^{2}+d_{j}^{-2}\left\|w_{h}\right\|_{0, D_{h}^{\prime}}^{2}\right)+C d_{j}^{-2}\left\|w_{h}\right\|_{0, D_{h}}^{2} \\
& \leqslant C\left(h_{j}^{2} d_{j}^{-2}\left\|w_{h}\right\|_{1, D_{h}^{\prime}}^{2}+d_{j}^{-2}\left\|w_{h}\right\|_{0, D_{h}^{\prime}}^{2}\right),
\end{aligned}
$$

from which an application of the inverse estimate (2.2) completes the proof of (4.3) and hence, of the lemma.

\section{Proofs of the Theorems and the Corollary.}

Proof of Theorem 1. Let $\tilde{u}$ be the approximate solution of (1.1) introduced in Section 3, and let $u_{h} \in S_{h}$ be the discrete solutions defined by (1.4). In view of Lemma 5 , since $\varepsilon=\operatorname{diam}\left(\tau_{0}\right)$ is of order $h^{1 / 1-\alpha)}$, it is sufficient for the proof of Theorem 1 to show that for the appropriate $\alpha$ 's

$$
\left\|\nabla\left(u_{h}-\tilde{u}\right)\right\|_{L_{1}} \leqslant C h^{r-1},
$$

and

$$
\left\|u_{h}-\tilde{u}\right\|_{L_{1}} \leqslant C h^{r}
$$

respectively.

For the proof, let $J \leqslant J_{1}$ be an integer such that $2^{-J}=C_{*} h^{1 / 1-\alpha)}$ for a suitably chosen sufficiently large constant $C_{*}$, to be determined later. Thus $d_{J}, h_{J}$, and $h^{1 / 1-\alpha)}$ are all of the same order, but $h_{J} d_{J}^{-1}=1 / C_{*}^{1-\alpha}$ can be made small by choosing $C_{*}$ large. Noting that by our definitions

$$
A\left(u_{h}-\tilde{u}, \chi\right)=0 \quad \forall \chi \in S_{h},
$$

we shall now prove first (5.1) and then (5.2).

Set $e=u_{h}-\tilde{u}$. We have at once, using Schwarz's inequality,

$$
\|\nabla e\|_{L_{1}}=\|\nabla e\|_{L_{1}\left(\Omega_{J+1}\right)}+\sum_{j \leqslant J}\|\nabla e\|_{L_{1}\left(D_{j}\right)} \leqslant C d_{J}^{N / 2}\|e\|_{1, \Omega_{j+1}}+C \sum_{j \leqslant J} d_{j}^{N / 2}\|e\|_{1, D_{j}} .
$$


The term associated with $\Omega_{J+1}$ can be appropriately estimated, for by Lemma 6 and (3.11) we have for $\alpha>(r-2) /(r-1)$,

$$
\begin{aligned}
d_{J}^{N / 2}\|e\|_{1} & \leqslant C d^{N / 2} h_{J}\|\tilde{\delta}\|_{0} \leqslant C d_{J}^{N / 2} h_{J} h^{\left.-\frac{1}{2} N / 1-\alpha\right)} \\
& =C C_{*}^{N / 2} h_{J}=C C_{*}^{N / 2+\alpha} h^{1 / 1-\alpha)} \leqslant C\left(C_{*}\right) h^{r-1} .
\end{aligned}
$$

Setting

$$
S=\sum_{j \leqslant J} d_{j}^{N / 2}\|e\|_{1, D_{j}}
$$

we shall show next that

$$
S \leqslant C h^{r-1}+\frac{1}{2} S \text {. }
$$

Hence it follows that $S \leqslant C h^{r-1}$, so that showing (5.4) completes the proof of (5.1).

As a matter of fact, we already have that

$$
S \leqslant C\left(C_{*}\right) h^{r-1}+\sum_{j<J} d_{j}^{N / 2}\|e\|_{1, D_{j}}
$$

We now make use of Lemma 7 to obtain

$$
\begin{aligned}
S & \leqslant C\left(C_{*}\right) h^{r-1}+C \sum_{j<J} d_{j}^{N / 2}\left(h_{j}^{r-1}\|\tilde{u}\|_{r, D_{j}^{1}}+d_{j}^{-1}\|e\|_{0, D_{j}^{1}}\right) \\
& \leqslant C\left(C_{*}\right) h^{r-1}+C \sum_{j \leqslant J} d_{j}^{N / 2} h_{j}^{r-1}\|\tilde{u}\|_{r, D_{j}}+C \sum_{j \leqslant J} d_{j}^{N / 2-1}\|e\|_{0, D_{j}} .
\end{aligned}
$$

By (3.6) and (3.12),

$$
\|\tilde{u}\|_{r, D_{j}} \leqslant C d_{j}^{-N / 2+2-r}\|\tilde{\delta}\|_{L_{1}} \leqslant C d_{j}^{-N / 2+2-r},
$$

so that for $\alpha>(r-2) /(r-1)$,

$$
\sum_{j \leqslant J} d_{j}^{N / 2} h_{j}^{r-1}\|\tilde{u}\|_{r, D_{j}} \leqslant C \sum_{j_{1} \leqslant j \leqslant J} d_{j}^{2-r} h_{j}^{r-1} \leqslant C h^{r-1} \sum_{j \geqslant j_{1}} d_{j}^{2-r+\alpha(r-1)} \leqslant C h^{r-1} .
$$

We have thus shown

$$
S \leqslant C\left(C_{*}\right) h^{r-1}+C_{2} \sum_{j \leqslant J} d_{j}^{N / 2-1}\|e\|_{0, D_{j}}
$$

where the last sum remains to be estimated.

For this purpose, let $e_{j}$ be of unit $L_{2}$-norm and such that $\|e\|_{0, D_{j}}=\left(e, e_{j}\right)$, with $\operatorname{supp}\left(e_{j}\right) \subset \bar{D}_{j}$, and let $v$ be the solution of

$$
A(\psi, v)=\left(\psi, e_{j}\right) \quad \forall \psi \in H^{1} .
$$

Hence $\|e\|_{0, D_{j}}=A(e, v)$, and owing to (5.3) (with $\chi=v_{I}$ ), the continuity of $A(\cdot, \cdot)$, and property (2.8) of the interpolant $v_{I}$,

$$
\begin{aligned}
\|e\|_{0, D_{j}} & =A\left(e, v-v_{I}\right) \\
& \leqslant C\|e\|_{1, \Omega_{J+1}}\left\|v-v_{I}\right\|_{1, \Omega_{J+1}}+C \sum_{i \leqslant J}\|e\|_{1, D_{i}}\left\|v-v_{I}\right\|_{1, D_{i}} \\
& \leqslant C h_{J}\|e\|_{1, \Omega_{J+1}}\|v\|_{2, \Omega_{J}}+C \sum_{i \leqslant J} h_{i}\|e\|_{1, D_{i}}\|v\|_{2, D_{i}^{1}}
\end{aligned}
$$


Employing the regularity estimates (3.2), (3.6), and (3.7), we obtain

$$
\begin{aligned}
\|e\|_{0, D_{j}} \leqslant & C h_{J} d_{J}^{N / 2} d_{j}^{-N / 2}\|e\|_{1, \Omega_{J+1}} \\
& +C d_{j}^{-N / 2} \sum_{j<i \leqslant J} h_{i} d_{i}^{N / 2}\|e\|_{1, D_{i}}+C d_{j}^{N / 2} \sum_{i \leqslant j} h_{i} d_{i}^{-N / 2}\|e\|_{1, D_{i}},
\end{aligned}
$$

and end up with

$$
\begin{aligned}
\|e\|_{0, D_{j}} \leqslant & C h_{J} d_{J}^{N / 2} d_{j}^{-N / 2}\|e\|_{1}+C d_{j}^{-N / 2} h_{j} \sum_{j<i \leqslant J} d_{i}^{N / 2}\|e\|_{1, D_{i}} \\
& +C d_{j}^{N / 2} \max _{i \leqslant j}\left(h_{i} d_{i}^{-N}\right) \sum_{i \leqslant j} d_{i}^{N / 2}\|e\|_{1, D_{i}} \\
\leqslant & C\left(C_{*}\right) h_{J} h^{r-1} d_{j}^{-N / 2}+C d_{j}^{-N / 2} h_{j} S .
\end{aligned}
$$

So, for $C_{*}$ sufficiently large, that is, for $h_{J} d_{J}^{-1}$ sufficiently small, we conclude

$$
\begin{aligned}
C_{2} \sum_{j \leqslant J} d_{j}^{N / 2-1}\|e\|_{0, D_{j}} & \leqslant C\left(C_{*}\right) h^{r-1} h_{J} \sum_{j \leqslant J} d_{j}^{-1}+C S \sum_{j \leqslant J} d_{j}^{-1} h_{j} \\
& \leqslant C\left(C_{*}\right) h^{r-1} h_{J} d_{J}^{-1}+C S h_{J} d_{J}^{-1} \leqslant C h^{r-1}+\frac{1}{2} S,
\end{aligned}
$$

which together with (5.6) shows (5.4) and completes the proof of (5.1).

We now turn to the proof of (5.2) and have at once

$$
\begin{aligned}
\|e\|_{L_{1}} & =\|e\|_{L_{1}\left(\Omega_{j+1}\right)}+\sum_{j \leqslant J}\|e\|_{L_{1}\left(D_{j}\right)} \\
& \leqslant C d_{J}^{N / 2}\|e\|_{0, \Omega_{J+1}}+C \sum_{j \leqslant J} d_{j}^{N / 2}\|e\|_{0, D_{j}} .
\end{aligned}
$$

As before, we first estimate the term associated with $\Omega_{J+1}$. Let $e_{J+1}$ have support in $\bar{\Omega}_{J+1}$, be of unit $L_{2}$-norm, and such that $\|e\|_{0, \Omega_{J+1}}=\left(e, e_{J+1}\right)$, and let $v$ solve

$$
A(\psi, v)=\left(\psi, e_{J+1}\right) \quad \forall \psi \in H^{1} \text {. }
$$

Using familiar arguments, we have

$$
\begin{aligned}
\|e\|_{0, \Omega_{J+1}} & =A\left(e, v-v_{I}\right) \leqslant C h_{J}\|e\|_{1, \Omega_{J+1}}\|v\|_{2, \Omega_{J}}+C \sum_{j \leqslant J} h_{j}\|e\|_{1, D_{j}}\|v\|_{2, D_{j}} \\
& \leqslant C h_{J}\|e\|_{1, \Omega_{J+1}}+C \sum_{j \leqslant J} h_{j}\|e\|_{1, D_{j}},
\end{aligned}
$$

where we have confined ourselves to use the standard regularity estimate (3.2) in the last step. In fact, we shall only need the weaker estimate

$$
d_{J}^{N / 2}\|e\|_{0, \Omega_{J+1}} \leqslant C J h_{J} d_{J}^{N / 2}\|e\|_{1, \Omega_{J+1}}+C \sum_{j \leqslant J} j h_{j} d_{j}^{N / 2}\|e\|_{1, D_{j}}
$$

where we assume, for notational simplicity, that $j_{1}>0$, that is, that $D_{j}$ is empty for $j \leqslant 0$. (Otherwise, the factors $j$ must be replaced by, say, $|j|+1$.) In order to see that the sum in (5.8) can be estimated in the same way, we apply the estimate (5.7) and change order of summation with respect to $j$ and $i$. We thus obtain

$$
\begin{aligned}
\sum_{j \leqslant J} d_{j}^{N / 2}\|e\|_{0, D_{j}} \leqslant & C h_{J} d_{J}^{N / 2}\|e\|_{1, \Omega_{J+1}} \sum_{j_{1} \leqslant j \leqslant J} 1 \\
& +C \sum_{i \leqslant J} h_{i} d_{i}^{N / 2}\|e\|_{1, D_{i}} \sum_{j_{1} \leqslant j<i} 1+C \sum_{i \leqslant J} h_{i} d_{i}^{-N / 2}\|e\|_{1, D_{i}} \sum_{i \leqslant j \leqslant J} d_{j}^{N} \\
\leqslant & C J h_{J} d_{J}^{N / 2}\|e\|_{1, \Omega_{J+1}}+C \sum_{i \leqslant J} i h_{i} d_{i}^{N / 2}\|e\|_{1, D_{i}},
\end{aligned}
$$


and by this we have shown

$$
\|e\|_{L_{1}} \leqslant C J h_{J} d_{J}^{N / 2}\|e\|_{1, \Omega_{J+1}}+C \sum_{j \leqslant J} j h_{j} d_{j}^{N / 2}\|e\|_{1, D_{j}} .
$$

At this point, in the case of $\alpha>(r-2) /(r-1)$, we obtain (5.2) by just applying our above estimates on $d_{J}^{N / 2}\|e\|_{1}$ and $S$. In order to prove (5.2) also for the smaller $\alpha$ 's we proceed from (5.10) as follows.

Once again we first estimate the term associated with $\Omega_{J+1}$. By Lemma 6, our assumption $\alpha>(r-2) / r$, and the fact that $J$ is of order $\ln 1 / h$, we have

$$
\begin{aligned}
J h_{J} d_{J}^{N / 2}\|e\|_{1} & \leqslant C J h_{J}^{2} d_{J}^{N / 2} h^{\left.-\frac{1}{2} N / 1-\alpha\right)} \leqslant C\left(C_{*}\right) J h_{J}^{2} \\
& \leqslant C\left(C_{*}\right) \ln \frac{1}{h} h^{2 \wedge 1-\alpha)} \leqslant C\left(C_{*}\right) h^{r} .
\end{aligned}
$$

Setting

$$
S^{\prime}=\sum_{j \leqslant J} j h_{j} d_{j}^{N / 2}\|e\|_{1, D_{j}}
$$

we shall now prove that

$$
S^{\prime} \leqslant C h^{r}+\frac{1}{2} S^{\prime}
$$

Since this yields $S^{\prime} \leqslant C h^{r}$, showing (5.11) completes the proof of (5.2).

Obviously,

$$
S^{\prime} \leqslant C\left(C_{*}\right) h^{r}+\sum_{j<J} j h_{j} d_{j}^{N / 2}\|e\|_{1, D_{j}},
$$

and in view of Lemma 7,

$$
S^{\prime} \leqslant C\left(C_{*}\right) h^{r}+\sum_{j \leqslant J} j h_{j}^{r} d_{j}^{N / 2}\|\tilde{u}\|_{r, D_{j}}+C \sum_{j \leqslant J} j h_{j} d_{j}^{N / 2-1}\|e\|_{0, D_{j}} .
$$

Using (5.5) and our assumption $\alpha>(r-2) / r$, we obtain

$$
\sum_{j \leqslant J} j h_{j}^{r} d_{j}^{N / 2}\|\tilde{u}\|_{r, D_{j}} \leqslant C \sum_{j \geqslant j_{1}} j h_{j}^{r} d_{j}^{2-r}=C h^{r} \sum_{j \geqslant j_{1}} j d_{j}^{r \alpha+2-r} \leqslant C h^{r},
$$

since the last sum is dominated by the convergent integral

$$
\int_{0}^{\infty}(x+1) 2^{-c x} d x, \quad c=r \alpha+2-r>0 .
$$

We have thus shown,

$$
S^{\prime} \leqslant C\left(C_{*}\right) h^{r}+C_{3} \sum_{j \leqslant J} j h_{j} d_{j}^{N / 2-1}\|e\|_{0, D_{j}},
$$

and we proceed to find an estimate also for the last sum.

Using (5.7) for a starting-point, we deduce that

$$
\begin{aligned}
\|e\|_{0, D_{j}} & \leqslant C h_{J} d_{J}^{N / 2} d_{j}^{-N / 2}\|e\|_{1, \Omega_{J+1}}+C j^{-1} d_{j}^{-N / 2} S^{\prime}+C d_{j}^{N / 2} \max _{j_{1} \leqslant i \leqslant j}\left(i^{-1} d_{i}^{-N}\right) S^{\prime} \\
& \leqslant C h_{J} d_{J}^{N / 2} d_{j}^{-N / 2}\|e\|_{1}+C j^{-1} d_{j}^{-N / 2} S^{\prime},
\end{aligned}
$$

where we stick to the convenient assumption $j_{1}>0$. For a suitable choice of $C_{*}$ we thus obtain

$$
\begin{aligned}
C_{3} \sum_{j \leqslant J} j h_{j} d_{j}^{N / 2-1}\|e\|_{0, D_{j}} & \leqslant C h_{J} d_{J}^{N / 2}\|e\|_{1} \sum_{j \leqslant J} j h_{j} d_{j}^{-1}+C S^{\prime} \sum_{j \leqslant J} h_{j} d_{j}^{-1} \\
& \leqslant C\left(C_{*}\right) J^{2} h_{J}^{2}+C S^{\prime} h_{J} d_{J}^{-1} \leqslant C h^{r}+\frac{1}{2} S^{\prime} .
\end{aligned}
$$


Joint with (5.12), this estimate shows (5.11) and hence completes the proof of (5.2) and of Theorem 1.

Proof of Theorem 2. In view of Corollary 5.1 of [13] and our assumptions, there are constants $c$ and $C$ such that for $d>c h^{1 / 1-\alpha)}$

$$
\left|u_{h}(x)-u(x)\right| \leqslant C\left(h d^{\alpha}\right)^{r}\left(\ln \left(h d^{\alpha-1}\right)\right)^{r}\|u\|_{W_{\infty}^{r}\left(B_{d / 2}(x)\right)}+C d^{-N}\left\|u_{h}-u\right\|_{L_{1}\left(B_{d / 2}(x)\right)},
$$

where we have used that the local mesh-size on $B_{d / 2}(x)$ is of order $h d^{\alpha}$. Together with (3.3) and our error estimate (1.8), since $\alpha>(r-2) / r$, this shows

$$
\begin{aligned}
\left|u_{h}(x)-u(x)\right| & \leqslant C h^{r}\left(\ln \left(h d^{\alpha-1}\right)\right)^{\bar{r}} d^{\alpha r-N+2-r}+C d^{-N}\left\|u_{h}-u\right\|_{L_{1}} \\
& \leqslant C d^{-N} h^{r}\left(\ln \frac{1}{h}\right)^{\bar{r}}
\end{aligned}
$$

which proves Theorem 2 .

It remains to demonstrate the

Proof of the Corollary. By the definitions of $v$ and $v_{h}$, we have

$$
A\left(\chi, v_{h}-v\right)=0 \quad \forall \chi \in S_{h},
$$

so that using also (1.3) and (1.4),

$$
v_{h}\left(x_{0}\right)-v\left(x_{0}\right)=A\left(u, v_{h}-v\right)=A\left(u-u_{h}, v_{h}-v\right)=A\left(u-u_{h}, v_{I}-v\right),
$$

where we take $v_{I} \in S_{h}$ to be the interpolant of $v$ defined in Lemma 2. By the continuity of $A(\cdot, \cdot)$, the error estimates of Theorem 1 , and the approximation property (2.9) of $v_{I}$, we thus have for $\alpha>(r-2) /(r-1)$,

$$
\left|v_{h}\left(x_{0}\right)-v\left(x_{0}\right)\right| \leqslant C\left\|u_{h}-u\right\|_{W_{1}^{1}}\left\|v_{I}-v\right\|_{W_{\infty}^{1}} \leqslant C h^{2 r-2}\|v\|_{W_{\infty}^{r}} .
$$

This proves the corollary.

6. Number of Elements. Construction of Refined Meshes. In this section, we shall first show that for $\alpha$ in $[0,1)$, the number of elements in the partitions $\Delta_{h}\left(x_{0}, \alpha\right)$ is of the same order, $O\left(h^{-N}\right)$, as in the corresponding quasi-uniform ones. Hence, the amount of work in solving the discrete variational problem (1.4) is not seriously increased by using our special spaces $S_{h}\left(x_{0}, \alpha, r\right)$ instead of standard finite element spaces. We shall then comment on the possibility of constructing meshes satisfying the crucial assumptions (1.5) and (1.6).

In order to estimate $N_{h}$, the total number of elements in $\Delta_{h}$, we first note that as a consequence of (1.5) there is a constant $c>0$, and for each $\tau \in \Delta_{h}$, an associated $j$, such that

$$
m\left(\tau \cap D_{j}\right) \geqslant c m(\tau) .
$$

For each $j$, we shall estimate the number $N_{j}$ of elements $\tau$ for which (6.1) holds and then sum over $j$. This will yield the desired estimate for $N_{h}$. It follows from (1.5) that if $\tau$ intersects $D_{j}$, then

$$
\operatorname{diam}(\tau) \geqslant C h d_{j}^{\alpha}
$$

and hence, by (1.6),

$$
m(\tau) \geqslant C h^{N} d_{j}^{N \alpha} .
$$

Since $m\left(D_{j}\right) \leqslant\left(2 d_{j}\right)^{N}$, we obtain

$$
N_{j} \leqslant C h^{-N} d_{j}^{N(1-\alpha)} .
$$


With $j_{1}$ as before and $\alpha$ in $[0,1)$, we thus find

$$
N_{h} \leqslant \sum_{j \geqslant j_{1}} N_{j} \leqslant C h^{-N} \sum_{j \geqslant j_{1}} d_{j}^{N(1-\alpha)} \leqslant C h^{-N}
$$

which proves our assertion.

We shall now see that a partition satisfying (1.5) and (1.6) can indeed be constructed. For notational simplicity, we consider the case $N=2$ and $x_{0}=0$. We first construct a (nontriangular) mesh of the correct mesh-size. Introduce polar coordinates $(r, \theta)$ and draw for the construction the circles $S_{i}$ of radii $r_{i}=(i h)^{1 / 1-\alpha)}$, $i=1,2, \ldots$. It follows that the distance between the two neighboring circles on distance $r_{i}$ and $r_{i+1}$ from 0 is approximately

$$
\left.h \frac{\partial}{\partial x} x^{1 / 1-\alpha)}\right|_{x=i h}=\frac{1}{1-\alpha} h(i h)^{\alpha \wedge 1-\alpha)}=\frac{1}{1-\alpha} h r_{i}^{\alpha},
$$

which is the mesh-size required in (1.5). In order to obtain the given mesh-size also in the $\theta$-direction we start with the rays $r \geqslant 0, \theta=2 \pi i / 4$ for $i=0, \ldots, 3$. We

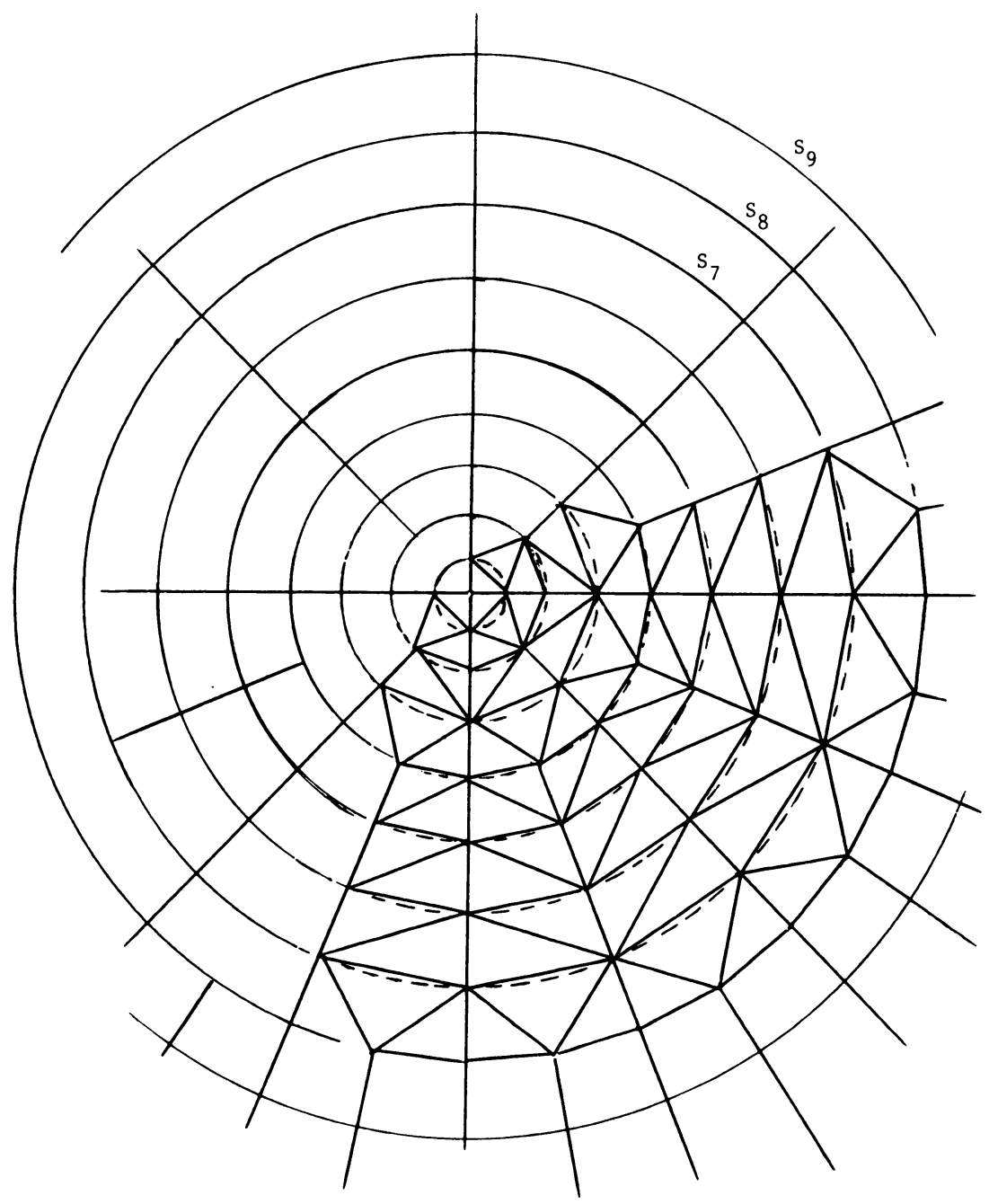

FIGURE 1 
then draw, for $m=1,2, \ldots$ and for $i=0, \ldots, 2^{m+1}-1$, the rays $r \geqslant r_{2} m, \theta=$ $(2 i+1) 2 \pi / 2^{m+2}$ as in Figure 1. In this way, for $2^{m}<i \leqslant 2^{m+1}$ the annulus between $S_{i-1}$ and $S_{i}$ is divided into $2^{m+2}$ equal pieces. Since the circumference of $S_{i}$ is $2 \pi r_{i}$ this implies that each such sector of an annulus is of width approximately

$$
2 \pi r_{i} / 2^{m+2} \sim r_{i} / i=h r_{i}^{\alpha}
$$

in the $\theta$-direction. Hence the partition so obtained satisfies (1.5). In order to modify this mesh into one consisting of triangles also satisfying (1.6) and the "face-to-face condition", we replace each sector of an annulus by a corresponding union of two or three triangles as shown in Figure 1, and the four sectors closest to 0 by the corresponding triangles. It is immediate that the triangles so obtained satisfy a minimum angle condition, which for interior elements is equivalent to (1.6). Once this is done it is easy, if needed, to modify the mesh so that (1.6) holds also for the boundary elements.

In higher dimensions a similar but somewhat more involved procedure is possible.

7. Appendix: an Extension Lemma. It is well known that functions in $W_{p}^{k}(\Omega)$ can be continuously extended into $W_{p}^{k}\left(R^{N}\right)$. In the proof of Lemma 2 of this paper, we use local estimates for such extensions. Therefore, we shall show below that by the method of extension using reflexions in the boundary (cf., e.g., Adams [1]), such estimates are easily obtained.

LEMMA A. Let $\Omega$ be a bounded domain in $R^{N}$ with smooth boundary $\Gamma$, and let $r$ be a nonnegative integer. Then there exist a linear operator $E: L_{1}(\Omega) \rightarrow L_{1}\left(R^{N}\right)$ and constants $C$ and $C_{E}$ such that $E v$ is an extension of $v$, that is, $E v=v$ on $\Omega$, and such that for $x \in \bar{\Omega}, d>0,1 \leqslant p \leqslant \infty, 0 \leqslant k \leqslant r$, and $v \in W_{p}^{k}\left(B_{C_{E} d}(x)\right)$, the estimate

$$
\|E v\|_{W_{p}^{k}\left(B_{d}(x)\right)} \leqslant C\|v\|_{W_{p}^{k}\left(B_{\left.C_{E^{d}}(x) \cap \Omega\right)}\right.}
$$

holds.

Proof. Given a set $D \subset R^{N}$, let us denote by $D^{+}\left(D^{-}\right)$the subset where $y_{N}>0$ $\left(y_{N}<0\right)$. In view of the assumed regularity of $\Gamma$ there exist a finite number of diffeomorphisms $\eta_{i}$ and a finite open cover $\left\{O_{i}\right\}_{i=1}^{M}$ of $\bar{\Omega}$ such that $\eta_{i}$ maps $O_{i}$ onto $B$ and $O_{i} \cap \Omega$ onto $B^{+}$, where $B$ is the unit ball $B_{1}(0)$. We shall first construct an extension operator $\tilde{E}: L_{1}\left(B^{+}\right) \rightarrow L_{1}(B)$ such that for $y \in \bar{B}^{+}, B_{d^{\prime}}(y) \subset B$, and $\tilde{v} \in W_{p}^{k}\left(B_{d^{\prime}}(y)^{+}\right)$

$$
\|\tilde{E} \tilde{v}\|_{W_{p}^{k}\left(B_{d^{\prime}}(y)\right)} \leqslant C\|\tilde{v}\|_{W_{p}^{k}\left(B_{d^{\prime}}(y)^{+}\right)},
$$

and then, using this case for reference, we define $E$ and prove (7.1).

With $y^{\prime}=\left(y_{1}, \ldots, y_{N-1}\right)$, set

$$
\tilde{E} \tilde{v}\left(y^{\prime}, y_{N}\right)= \begin{cases}\tilde{v}\left(y^{\prime}, y_{N}\right) & \text { for } y_{N}>0 \\ \sum_{j=1}^{r} m_{j} \tilde{v}\left(y^{\prime},-y_{N} / j\right) & \text { for } y_{N}<0\end{cases}
$$

where the coefficients $m_{j}$ are determined by the linearly independent equations

$$
\sum_{j=1}^{r} m_{j}(-1 / j)^{k}=1 \text { for } k=0,1, \ldots, r-1 \text {. }
$$


It is sufficient to prove (7.2) for smooth $\tilde{v}$ 's. But in that case, in view of the regularity of the extension across $y_{N}=0$, we have for $k \leqslant r$,

$$
\|\tilde{E} \tilde{v}\|_{W_{p}^{k}\left(B_{d^{\prime}}(y)\right)}=\|\tilde{E} \tilde{v}\|_{W_{p}^{k}\left(B_{d^{\prime}}(y)^{+}\right)}+\|\tilde{E} \tilde{v}\|_{W_{p}^{k}\left(B_{d^{\prime}}(y)^{-}\right)},
$$

and hence (7.2) follows at once. Now, let $\left\{\beta_{i}\right\}_{i=1}^{M}$ be a partition of unity on $\bar{\Omega}$ subject to $\operatorname{supp}\left(\beta_{i}\right) \subset O_{i}$ for $1 \leqslant i \leqslant M$, and set

$$
E v=\sum_{j=1}^{M} \beta_{i}\left(\tilde{E}\left(v \circ \eta_{i}^{-1}\right) \circ \eta_{i}\right)
$$

Obviously, this defines $E$ as a linear extension operator. In order to prove (7.1) we first restrict ourselves to the case of suitably small $d$ 's and estimate each term in (7.3). With $i$ fixed, we may then assume that $B_{d}(x)$ intersects $\operatorname{supp}\left(\beta_{i}\right)$ (otherwise there is nothing to prove) and that $B_{d}(x) \subset O_{i}$, since $\operatorname{supp}\left(\beta_{i}\right)$ is a compact subset of $O_{i}$. Let $y=\eta_{i}(x)$ and let $B_{d^{\prime}}(y)$ be the ball of smallest radius containing $\eta_{i}\left(B_{d}(x)\right)$. By the smoothness of $\eta_{i}$ and $\eta_{i}^{-1}$ we may further assume that for small $d$ 's, $B_{d^{\prime}}(y) \subset B$ and that there is a constant $C_{i}$ such that $\eta_{i}^{-1}\left(B_{d^{\prime}}(y)\right) \subset B_{C_{i} d}(x)$. But then

$$
\begin{aligned}
\left\|\beta\left(\tilde{E}\left(v \circ \eta_{i}^{-1}\right) \circ \eta_{i}\right)\right\|_{W_{p}^{k}\left(B_{d}(x)\right)} & \leqslant C\left\|\tilde{E}\left(v \circ \eta_{i}^{-1}\right)\right\|_{W_{p}^{k}\left(B_{d^{\prime}}(y)\right)} \\
& \leqslant C\left\|v \circ \eta_{i}^{-1}\right\|_{W_{p}^{k}\left(B_{d^{\prime}}(y)^{+}\right)} \leqslant C\|v\|_{W_{p}^{k}\left(B_{C_{i}}(x) \cap \Omega\right)} .
\end{aligned}
$$

Hence, (7.1) follows with $C_{E}=\max _{i} C_{i}$. For the smaller $d$ 's, this completes the proof. Since we may as well assume that $E v$ vanishes outside any given neighborhood of $\bar{\Omega}$, the estimates (7.1) for the larger $d$ 's follow from what we have proved for the smaller $d$ 's by an obvious covering argument. This completes the proof.

Department of Mathematics

Chalmers University of Technology and

The University of Göteborg

S-412 96 Göteborg, Sweden

1. R. A. Adams, Sobolev Spaces, Academic Press, New York, 1975.

2. I. BABUSKA, "Error bounds for the finite element method," Numer. Math., v. 16, 1971, pp. 322-333.

3. I. BABUSKA \& A. K. AzIz, "Survey lectures on the mathematical foundations of the finite element method," Section 6.3.6 of The Mathematical Foundations of the Finite Element Method with Applications to Parital Differential Equations, (A. K. Aziz, ed.), Academic Press, New York, 1973.

4. J. H. Bramble \& S. R. Hilbert, "Estimation of linear functionals on Sobolev spaces with application to Fourier transforms and spline interpolation," SIAM J. Numer. Anal., v. 7, 1970, pp. $112-124$.

5. J. H. Bramble \& A. H. Schatz, "Estimates for spline projections," RAIRO Anal. Numér., v. 10, 1976, pp. 5-37.

6. P. G. Ciarlet, The Finite Element Method for Elliptic Problems, North-Holland, Amsterdam, 1978.

7. P. G. Ciarlet, "Discrete variational Green's function. I," Aequationes Math., v. 4, 1970, pp. 74-82.

8. K. ERIKSSON, Improved Convergence by Mesh-Refinement in the Finite Element Method, Thesis, Chalmers University of Technology and the University of Göteborg, 1981.

9. S. R. Hilbert, "A mollifier useful for approximations in Sobolev spaces and some applications to approximating solutions of differential equations," Math. Comp., v. 27, 1973, pp. 81-89.

10. JU. P. KRASOVSKII, "Isolation of singularities of the Green's function," Math. USSR-Izv., v. 1, 1967, pp. 935-966. 
11. J. L. Lions \& E. Magenes, Problèmes aux Limites Non Homogènes et Applications, Vol. 1, Dunod, Paris, 1968.

12. J. A. Nitsche \& A. H. Schatz, "Interior estimates for Ritz-Galerkin methods," Math. Comp., v. 28,1974 , pp. 937-958.

13. A. H. SChATZ \& L. B. WAHLBIN, "Interior maximum norm estimates for finite element methods", Math. Comp., v. 31, 1977, pp. 414-442.

14. R. Scotr, “Finite element convergence for singular data," Numer. Math., v. 21, 1973, pp. 317-327.

15. R. Scorr, "Optimal $L_{\infty}$ estimates for the finite element method on irregular meshes," Math. Comp., v. 30, 1976, pp. 681-697. 